\title{
Corticotropic and serotonergic responses to acute stress with/without prior exercise training in different rat strains
}

\author{
C.L. MARTIN, ${ }^{1}$ M. DUCLOS,${ }^{1}$ S. AGUERRE, ${ }^{2}$ P. MORMEDE, ${ }^{2}$ G. MANIER ${ }^{1}$ \\ and F. CHAOULOFF ${ }^{2}$ \\ 1 Laboratoire de Pbysiologie de l'Exercice Musculaire et du Sport, Universite' Bordeaux II, 146 rue Léo Saignat, Bordeaux Cedex, \\ France \\ 2 Laboratoire Neurogénétique et Stress, INSERM U471, Institut Franfois Magendie, Université Bordeaux II, Bordeaux Cedex, \\ France
}

\begin{abstract}
The ability to cope with exercise training depends both on environmental and genetic background; however, whether the genetic status may affect (i) the hormonal status of trained subjects and, (ii) its responses to a heterotypic stressor is unknown. Herein, we have used Spontaneously Hypertensive Rats (SHR) and Lewis rats, that differ with regard to their psychoneuroendocrine profiles, to study the influences of an 8-week training programme and/or a 1-h immobilization stress on plasma adrenocorticotropin (ACTH) and corticosterone levels. In addition, brain serotonin metabolism was also measured as an index of neurochemical reactivity to stress. The amplitude of immobilizationelicited increases in ACTH levels which differed with the rat strain (Lewis > SHR), was amplified by prior training; besides, training decreased the strain difference in basal corticosterone (SHR > Lewis) and affected corticosterone response to immobilization in a strain-dependent manner. Thus, immobilization, which increased corticosterone levels in sedentary Lewis but not in SHRs, did not reveal interstrain differences in trained rats. Taken with the observation of a stimulatory effect of training on adrenal weights in SHRs, but not in Lewis, it is concluded that the effects of training on the corticotropic axis depend on the genetic profile of the individual. Lastly, training amplified the response of midbrain (but not striatum or hippocampus) serotonin metabolism to immobilization in a strain-independent manner although the levels of serotonin precursor, namely tryptophan, varied with training and immobilization in a strain-dependent manner. This study shows that some neuroendocrine and neurochemical effects of training undergo interindividual variability.
\end{abstract}

Keywords ACTH, corticosterone, exercise training, immobilization, Lewis rats, serotonin, SHR.

Received 16 April 1999, accepted 26 November 1999

Endurance training leads to cardiovascular (Kregel et al. 1990), metabolic (Henriksson et al. 1985), and hormonal adaptations (Watanabe et al. 1991, Duclos et al. 1996) so that there is a minimal homeostatic disruption in the exercising human or animal. Actually, studies in humans and animals have demonstrated that endurance capacity has a heritable component (Hagberg et al. 1998, Koch et al. 1998). In rats, Koch et al. (1998) have reported that $39 \%$ of the variation in running endurance performance between low and high performing Sprague-Dawley tats were determined by heritable factors. Confirming this, a recent study using 11 inbred rats strains reported a genetic influence on running capacity on a treadmill (Barbato et al. 1998).
The hypothalamo-pituitary-adrenal (HPA) axis is both stimulated by muscle exercise and subjected to adaptation with endurance training (Luger et al. 1987). Again, genetic differences in HPA axis activity have been demonstrated in humans (Petrides et al. 1997). In rodents, among different inbred rat strains, Lewis rats exhibit impaired corticotropin-releasing hormone (CRH), ACTH and corticosterone (CORT) responses to physical and emotional stressors whereas. Fisher 344 rats are characterized by their exquisitely sensitive and potent HPA axis hormonal responsiveness to these stressors (Sternberg et al. 1992). As far as the influence of genetic factors on HPA axis activity is concerned, the question of the relevance of the genetic background 
in the capacity to produce adaptations with chronic physical training may arise. Using treadmill exercise 5 days a week for 6-12 weeks, protocols of endurance training have emerged in rats (Armstrong et al. 1983). Unfortunately, all these endurance training programmes have been conducted with standard rat strains (Wistar, Sprague-Dawley). Another paradigm to define the factors that may modulate the physiological impact of exercise training is to take advantage of rat strains differing genetically in their pattern of neuroendocrine responses to different stressors. Moreover, as it may appear difficult to ascertain if training-induced differentiation in neuroendocrine responses would have emerged on the basis of a standard endocrine screening, another approach is to examine the effect of exercise training on the HPA axis response elicited by a further acute heterotypic stress. Data on the putative influence of the genetic factors on neuroendocrine responses to acute stress following endurance training are however, presently lacking.

In keeping with the aforementioned effect of the genetic background on endurance capacity and HPA axis responses to different stressors, we have analysed the neuroendocrine responses to acute stress (immobilization) following 8 weeks of exercise training in two different rat strains, namely the Spontaneously Hypertensive Rat (SHR) strain and the Lewis rat strain. The SHRs and Lewis rats were selected on the basis of their respective corticotropic responses to stressors that differ in intensity and type. Following a forced swimming stress (Armario et al. 1995), SHR and Lewis strains display, respectively, the highest stress-induced hyperglycaemia, and the lowest ACTH and CORT responses to this stress. Moreover, using chronic immobilization stress, Gomez et al. (1996) observed interstrain differences in ACTH and CORT levels, with a CORT hyporesponsiveness of Lewis rats to chronic immobilization.

Finally, both endurance training and acute immobilization affect different systems of central neurotransmitters. Among these neurotransmitters, serotonin (5-hydroxytryptamine, 5-HT) seems sensitive to both types of stress (Chaouloff 1993). Actually, stressinduced changes in 5-HT synthesis/metabolism relate to peripheral and central mechanisms (see below), thus indicating the usefulness of 5-HT synthesis/metabolism assessments as indices of the individual's sensitivity to stress.

The objective of the present study was thus to determine how the neuroendocrine effects of chronic exercise are affected by the genetic status of the animals. For this purpose, SHR and Lewis rats were submitted to an 8-week treadmill exercise protocol to induce an exercise training effect. At the end of this exercise training programme, an acute stress was further achieved by submitting rats to an $1-\mathrm{h}$ immobilization stress. Animals were then screened for (i) their body weight, (ii) the activity of their HPA axis (evaluated by measuring adrenal weight, plasma ACTH and CORT) and (iii) their 5-HT synthesis/metabolism rates in the midbrain, the striatum and the hippocampus.

\section{MATERIALS AND METHODS}

\section{Animals}

Experiments were conducted with 32 male SHRs and 32 male Lewis rats (IFFA CREDO, Les Oncins, France). The animals, 3 weeks old on arrival at our laboratory, were housed by strain 4 /cage (with food and water $a d$ libitum, except during the running sessions) in an animal quarter under constant temperature and a $12 \mathrm{~h} / 12 \mathrm{~h}$ light-dark cycle (lights on at 07.00 hours). Rats were left undisturbed for 1 week before the beginning of the experiments. The animals were weighed once a week (initial weight: Lewis: $171 \pm 7 \mathrm{~g}$ and SHRs: $135 \pm 10 \mathrm{~g}, P<0.01$ ). This study was conducted in conformity with the French publication on animal experimentation.

\section{Experimental design}

The animals were randomly assigned to either a trained (Lewis: $n=16$, SHRs: $n=16$ ) or untrained (Lewis: $n=16$, SHRs: $n=16$ ) group. The trained group underwent 8 weeks of exercise training, which was performed on a horizontal treadmill, containing four running compartments, and driven by an electrical motor (Chaouloff et al. 1985). Rats ran 5 days week ${ }^{-1}$, $1 \mathrm{~h} \mathrm{day}^{-1}$. In order to minimize stress, treadmill training began by familiarizing the rats to the apparatus for 1 week (five sessions, 5-20 min at a speed of 15-18 $\mathrm{m} \mathrm{min}^{-1}$ ), electrical foot shocks being initially delivered when the rats stepped down from the treadmill. Throughout the study, each rat tan in the same compartment. After this first adaptation week, treadmill speed was gradually increased from 18 to $25 \mathrm{~m} \mathrm{~min}^{-1}$ (slope at $0^{\circ}$ ) at the end of training, electrical foot shocks not being delivered anymore. The animals exercised in the morning (08.00-12.00 hours). Meanwhile, animals belonging to the untrained group were removed from their respective cages and gently placed in a novel cage.

Two days after the completion of these 8 weeks of training, each group (trained and untrained) was further divided into two groups. Animals were weighed, and then either underwent immobilization stress (first group), or remained in their home cage (second group: controls). This stress consisted in $1 \mathrm{~h}$ of immobilization in plastic restrainers (Chaouloff et al. 1994). Rats from the two groups were successively killed by decapitation 
in an adjacent room (09.00-12.00 hours). Trunk blood was collected in $10-\mathrm{mL}$ polyethylene tubes containing $0.1 \mathrm{~mL}$ of $10 \%$ EDTA, and stored in ice until centrifugation. Immediately after decapitation, rat brains were rapidly removed. Brain regions (midbrain, striatum, hippocampps) on the one hand, and adrenals on another hand, were dissected and stored at $-80^{\circ} \mathrm{C}$ until analysis.

\section{Analysis of circulating ACTH and corticosterone}

Plasma ACTH was assayed immunoradiometrically with the use of a commercially available kit using $\left[{ }^{125}\right] \mathrm{ACTH}$ as the tracer (Nichols Institute Diagnostics, San Juan Capistrano, USA). Plasma corticosterone was measured after alcohol extraction by a competitive protein binding assay (Chaouloff et al. 1995) using rhesus monkey serum as the source of transcortin, $\left[{ }^{3} \mathrm{H}\right]$ corticosterone as the tracer, and dextran-coated charcoal to absorb the unbound fraction. Samples were analysed in duplicate.

\section{Tryptophan (Tpp), 5-HT and 5-bydroxyindoleacetic acid (5-HLAA) analysis}

Frozen midbrains, hippocampus and striati were homogenized by sonication in $0.4 \mathrm{~N}$ perchloric acid (Chaouloff et al. 1985). The resulting homogenate was centrifuged $10 \mathrm{~min}$ at $14000 \times \mathrm{g}$. From the supernatant, $25 \mu \mathrm{L}$ were kept for the measurement of tissue $\operatorname{Trp}$, 5-HT, and 5-HIAA concentrations by high performance liquid chromatography (HPLC) coupled either to an electrochemical detector $(650 \mathrm{mV}: 5-\mathrm{HT}$ and 5-HIAA) or to an ultraviolet detector (280 nm: Trp). The mobile phase was a sodium phosphate buffer (50 nM, pH 3.3) containing 1-octanesulphonic acid $\left(100 \mathrm{mg} \mathrm{L}^{-1}\right)$ EDTA $\left(150 \mathrm{mg} \mathrm{L}^{-1}\right)$ and methanol $(15 \%)$, as already described (Kulikov et al. 1997). For plasma $\operatorname{Trp}$ analysis, samples were mixed with $0.4 \mathrm{~N}$ perchloric acid, centrifuged, and $25 \mu \mathrm{L}$ of the supernatant injected into the HPLC system. Trp was then measured as described above.

\section{Statistics}

Data are presented as mean $\pm \mathrm{SE}$. Body weight evolution was analysed by means of a two-way analysis of variance (ANOVA) with repeated measures. Absolute body weights and adrenal weights were analysed by means of two-way ANOVAs with strain (two levels) and training (two levels) as between-subject factors. All other data were analysed by means of a three-way ANOVA with strain (two levels), training (two levels) and immobilization (two levels) as between-subject factors. When significant, analyses of variance were followed by post hoc individual comparisons (Newman-Keuls test).

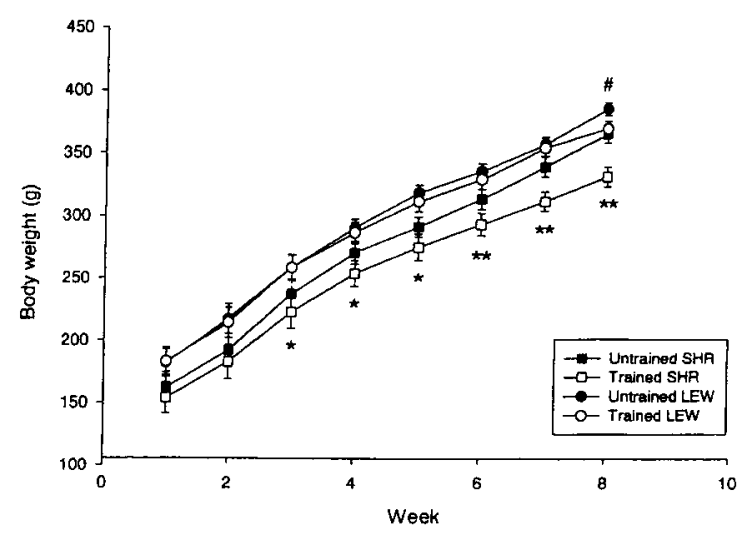

Figure 1 Body weight of untrained and trained Spontaneously Hypertensive Rats (SHR) and Lewis rats (LEW). Results are expressed as mean $\pm \mathrm{SE}$ of 16 rats. $* P<0.05, * * P<0.01$ for the differences between untrained and trained SHRs; $\# P<0.05$ for the differences between untrained and trained Lewis rats.

\section{RESULTS}

\section{Effects of training on body and adrenal weights}

Figure 1 shows time-dependent influence of training on the respective body weights of SHRs and Lewis rats. Compared with their untrained counterparts, trained Lewis rats did not show any body weight effect of training excepted during the last week of the experiment, when untrained Lewis weighed significantly more than their trained counterparts $(P<0.05)$. By contrast, trained SHRs had significantly lower body weights than untrained SHRs from the third week of training $(P<0.05)$. This difference grew up until the end of the experiment so that untrained SHRs were $11 \%$ heavier than trained SHRs whereas in Lewis rats that difference reached only $4 \%$.

At the end of the experiment (on the day of the sacrifice), untrained and trained Lewis displayed heavier mean body weights compared with their respective untrained and trained SHRs counterparts $\left(F_{1,60}=19.53, P<0.001\right.$ ) (Table 1). However, post hoc analyses revealed that such a difference reached significance only in trained rats $(P<0.01)$. Beside, training per se resulted in a significant decrease of body weight in both strains $\left(F_{1,60}=13.74, P<0.001\right)$.

Absolute adrenal weights were heavier in SHRs than in Lewis rats $\left(F_{1,59}=17.78, P<0.001\right)$ with this strain difference reaching significance only in trained rats (Table 1). When expressed as the ratio over body weights, the (relative) adrenal weight of SHRs was increased, compared with Lewis rats $\left(F_{1,59}=41.7\right.$, $P<0.001)$ both in untrained $(P<0.05)$ and trained conditions $(P<0.01)$. In addition, a strain $\times$ training interaction $\left(F_{1,59}=4.18, P<0.05\right)$ could be noted with a significant relative adrenal weight increase in 


\begin{tabular}{|c|c|c|c|}
\hline & $\begin{array}{l}\text { Body weight } \\
\text { (g) }\end{array}$ & $\begin{array}{l}\text { Adrenal weight } \\
\text { (mg) }\end{array}$ & $\begin{array}{l}\text { Relative adrenal weight } \\
\left(\mathrm{mg} 100 \mathrm{~g}^{-1}\right)\end{array}$ \\
\hline SHR untrained & $366 \pm 7$ & $47 \pm 2$ & $13.01 \pm 0.53$ \\
\hline LEW untrained & $386 \pm 5$ & $42 \pm 2$ & $10.80 \pm 0.36^{\#}$ \\
\hline SHR trained & $332 \pm 8^{* *}$ & $52 \pm 2$ & $15.71 \pm 0.55^{* *}$ \\
\hline LEW trained & $371 \pm 6^{* *} \# \#$ & $42 \pm 2^{\# \#}$ & $11.45 \pm 0.54^{\# \#}$ \\
\hline Strain effect & + & + & + \\
\hline Training effect & + & & + \\
\hline Strain $\times$ training interaction & & & + \\
\hline
\end{tabular}

Table 1 Final total body weight, adrenal weight, and relative adrenal weight of untrained and trained SHRs and Lewis rats

Results are expressed as mean $\pm \mathrm{SE}$ of 16 rats. $+P<0.05$ for the ANOva results. $\# P<0.05$ and $\# \# P<0.01$ for the differences with SHRs; ${ }^{* *} P<0.01$ for the differences with untrained rats.

SHRs, but not in Lewis rats with training $(+18 \%$, $P<0.01$ )

Effects of training and/or immobilization on the HPA axis

Training $\left(F_{1,51}=4.60, P<0.05\right)$ and immobilization $\left(F_{1,51}=86.09, P<0.01\right)$ were endowed with significant effects (Fig. 2a). Besides, strain $X$ immobilization $\left(F_{1,51}=4.34, P<0.05\right)$ and training $\times$ immobilization $\left(F_{1,51}=5.02, P<0.05\right)$ interactions could be noted. Thus, trained immobilized Lewis showed higher ACTH plasma concentration compared with their respective SHR counterparts whereas only trained immobilized Lewis showed higher ACTH concentration compared with their untrained counterparts $(P<0.01)$. With regard to the training $X$ immobilization interaction, trained rats displayed a greater immobilization-induced increase in ACTH levels, compared with untrained rats.

As Fig. 2(b) shows, the ANOva revealed a strain effect $\left(F_{1,50}=11.30, P<0.005\right)$ on basal CORT levels with untrained control SHRs showing higher plasma CORT values than their Lewis counterparts $(300+40$ vs. $42+12 \mathrm{ng} \mathrm{mL}^{-1}, P<0.01$ ). For trained control animals, the amplitude of the difference decreased, but remained significant $(P<0.05)$. In addition, immobilization induced a significant increase in plasma CORT concentration $\left(F_{1,50}=56.29, P<0.001\right) \quad$ (Fig. 2b). However, post hoc analysis revealed no significant immobilization-induced plasma CORT increase in untrained SHRs compared with their trained counterparts, whereas in untrained Lewis rats, a marked immobilization-induced CORT increase was noticed (+897\%). Lastly, training was devoid of any effect per se.

The corticosterone/ACTH ratio is presented in Fig. 2(c). The SHR strain displayed a significantly higher adrenal sensitivity to ACTH, compared with the Lewis strain $\left(F_{1,49}=16.66, P<0.001\right)$, with exercise training bearing no effect. When immobilized, both SHRs and Lewis rats showed decreased adrenal sensitivity $\left(F_{1,49}=20.58, P<0.001\right)$; however, post hoc analyses revealed a significant decrease between control and immobilized SHRs, whereas Lewis rats did not.
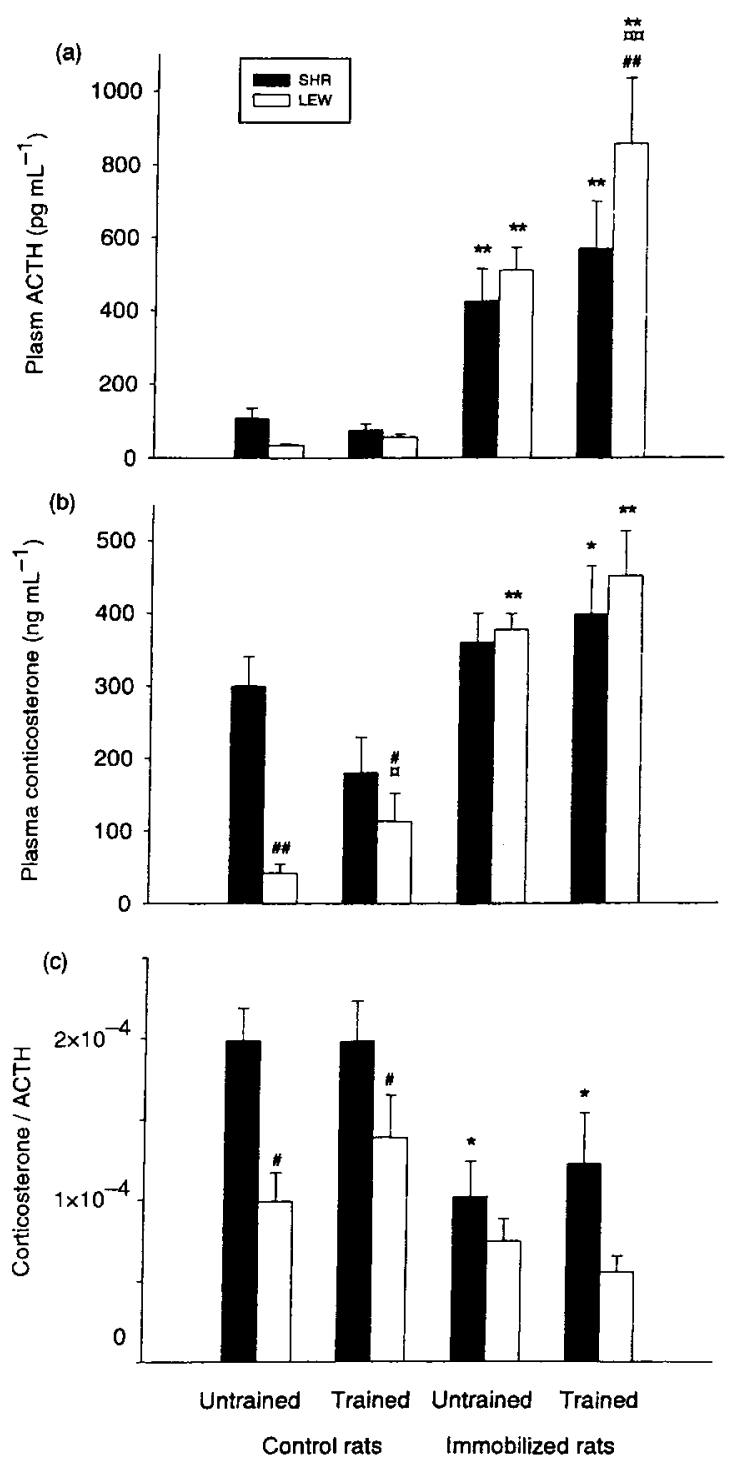

Figure 2 Plasma ACTH (a) and corticosterone (b) levels and corticosterone/ACTH ratio (c) in untrained and trained Spontaneously Hypertensive Rats (SHR) and Lewis rats (LEW) (control or $1 \mathrm{~h}$ of immobilization stress). Results are expressed as means $\pm \mathrm{SE}$ of eight rats. $\# P<0.05$ and \#\#P<0.01 for the differences with SHRs; $* P<0.05$ and $* * P<0.01$ for the differences with the control rats; $P<0.05$ and $P<0.01$ for the differences with untrained rats. 

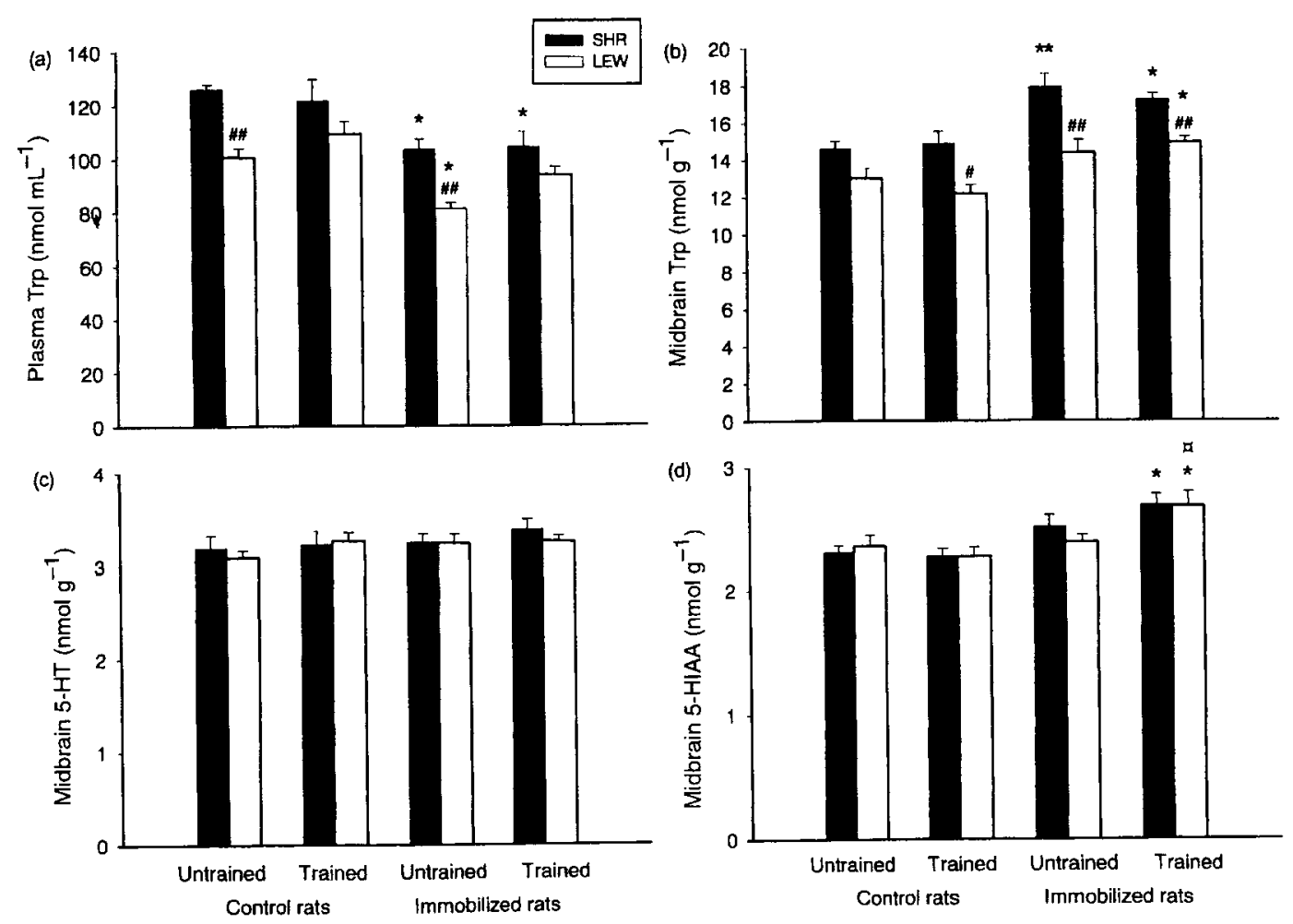

Figure 3 Plasma tryptophan ( $\operatorname{Trp}$ ) (a) and midbrain Trp (b), 5-hydroxytryptamine (5-HT) (c) and 5-hydroxyindoleacetic acid (5-HIAA) (d) levels in untrained and trained Spontaneously Hypertensive Rats (SHR) and Lewis rats (LEW) (control or $1 \mathrm{~h}$ of immobilization stress). Results are expressed as mean $\pm \mathrm{SE}$ of eight rats. $\# P<0.05$ and $\# \# P<0.01$ for the differences with SHRs; $* P<0.05$ and $* * P<0.01$ for the differences with the control rats; $P<0.05$ for the differences with untrained rats.

\section{Serotonergic metabolism}

As shown in Fig. 3(a), SHRs displayed higher total plasma Trp compared with Lewis rats $\left(F_{1,47}=28.75\right.$, $P<0.01)$. A strain $\times$ training interaction $\left(F_{1,47}=4.25\right.$, $P<0.05)$ revealed that the amplitude of such a difference decreased in trained rats. Indeed, plasma $\operatorname{Trp}$ tended to decrease with training in the SHR strain, whereas it tended to increase in the Lewis one: actually, this strain difference reached post hoc significance in untrained rats only $(P<0.01)$. Lastly, immobilization stress decreased total plasma Trp levels under all experimental conditions $\left(F_{1,47}=32.49, P<0.01\right)$.

Table 2 and Fig. 3 show the influence of strain, training and/or immobilization on midbrain, hippocampal and striatum levels of Trp, 5-HT and 5-HIAA. Trp concentrations increased with immobilization in midbrain $\left(F_{1,55}=37.81, \quad P<0.001\right)$, hippocampus $\left(F_{1,55}=33.81, P<0.001\right)$ and striatum $\left(F_{1,55}=48.39\right.$, $P<0.001)$; beside, a strain effect could be noted with SHRs displaying higher Trp concentrations than Lewis rats in the three aforementioned brain regions $\left(F_{1,55}=40.02, P<0.001 ; F_{1,55}=59.19, P<0.001\right.$; $F_{1,55}=49.72, P<0.001$, respectively) (Table 2 and Fig. 3b). Alternatively, training was devoid of any effect, either per se or in the interaction with the two main variables.

With regard to 5-HT, the sole effect that was observed was a strain effect in the striatum $\left(F_{1,55}=4.47, P<0.05\right)$ where Lewis rats displayed slightly higher levels of the amine than in SHRs (Table 2). Neither the strain nor the training affected central 5-HIAA levels; on the other hand, immobilization was endowed with a stimulatory influence in midbrain $\left(F_{1,55}=20.92, \quad P<0.01\right)$, hippocampus $\left(F_{1,55}=16.36, P<0.001\right)$ and striatum $\left(F_{1,55}=48.56\right.$, $P<0.001$ ) (Table 2 and Fig. 3d). In midbrain, this stimulatory effect of immobilization was particularly evident in trained animals $\left(F_{1,55}=6.35, P<0.05\right.$ for the training $\times$ immobilization interaction; Fig. $3 \mathrm{~d}$ ) whereas in the two other brain regions a strain $x$ training interaction could be noted $\left(F_{1,55}=6.40\right.$, $P<0.05 ; F_{1,55}=4.40, P<0.05$, in hippocampus and striatum, respectively). Thus, in the hippocampus of untrained rats, 5-HIAA levels tended to be higher in SHRs than in Lewis rats, an observation that did not extend to trained animals (Table 2). On the contrary, in striatum, 5-HIAA levels tended to be higher in untrained Lewis rats than in untrained SHRs whereas the converse was true for trained animals (Table 2). 
Table 2 Effects of an 8-week treadmill training and/or 1 h of immobilization stress ( $(\mathrm{mm})$ on the concentrations (nmol $\mathrm{g}^{-1}$ ) of tryptophan (Trp), 5-hydroxytryptamine (5-HT) and 5-hydroxyindoleacetic acid (5-HIAA) in the hippocampus and striatum of SHRs and Lewis rats

\begin{tabular}{|c|c|c|c|c|c|c|}
\hline \multirow[b]{2}{*}{ Group } & \multicolumn{3}{|l|}{ Hippocampus } & \multicolumn{3}{|l|}{ Striatum } \\
\hline & $\operatorname{Trp}$ & 5-HT & 5-HIAA & $\operatorname{Trp}$ & 5-HT & 5-HIAA \\
\hline SHR untrained control & $17.98 \pm 0.41$ & $1.39 \pm 0.07$ & $1.40 \pm 0.10$ & $20.10 \pm 0.66$ & $1.77 \pm 0.08$ & $1.44 \pm 0.05$ \\
\hline LEW untrained control & $14.68 \pm 0.75$ & $1.46 \pm 0.12$ & $1.22 \pm 0.05$ & $17.29 \pm 0.57$ & $2.17 \pm 0.08$ & $1.52 \pm 0.06$ \\
\hline SHR trained control. & $18.94 \pm 0.87$ & $1.45 \pm 0.09$ & $1.23 \pm 0.05$ & $20.04 \pm 0.69$ & $2.00 \pm 0.11$ & $1.48 \pm 0.08$ \\
\hline LEW trained control & $14.17 \pm 0.53^{\# \#}$ & $1.35 \pm 0.09$ & $1.21 \pm 0.04$ & $16.65 \pm 0.25^{\#}$ & $2.04 \pm 0.10$ & $1.39 \pm 0.04$ \\
\hline SHR untrained Imm & $21.60 \pm 0.89^{*}$ & $1.51 \pm 0.07$ & $1.51 \pm 0.07$ & $23.18 \pm 0.70^{*}$ & $2.03 \pm 0.04$ & $1.71 \pm 0.04$ \\
\hline LEW untrained Imm & $16.43 \pm 0.53^{\# \#}$ & $1.55 \pm 0.14$ & $1.40 \pm 0.07$ & $19.65 \pm 0.45^{\# \#}$ & $1.97 \pm 0.10$ & $1.87 \pm 0.10^{*}$ \\
\hline SHR trained Imm & $21.82 \pm 1.17$ & $1.47 \pm 0.16$ & $1.36 \pm 0.06$ & $23.97 \pm 1.25^{* *}$ & $1.91 \pm 0.10$ & $1.82 \pm 0.10^{*}$ \\
\hline LEW trained Imm & $18.46 \pm 0.71^{* *}$ & $1.57 \pm 0.13$ & $1.56 \pm 0.07^{*}$ & $20.40 \pm 0.48^{* * *}$ & $2.05 \pm 0.08$ & $1.76 \pm 0.03^{* *}$ \\
\hline 1 - Strain effect & + & & & + & + & \\
\hline \multicolumn{7}{|l|}{$2-$ Training effect } \\
\hline 3 - Immobilization effect & + & & + & + & & + \\
\hline Interaction effect & & & $1 \times 2$ & & $1 \times 2 \times 3$ & $1 \times 2$ \\
\hline
\end{tabular}

Results are expressed as mean $\pm \mathrm{SE}$ of eight rats. $+P<0.05$ for the ANOva results. $\# P<0.05$ and \#\# $P<0.01$ fot the differences with SHRs; $* P<0.05$ and $* * P<0.01$ for the differences with the control rats.

\section{DISCUSSION}

\section{Baseline differences between untrained SHRs and Lewis rats}

Absolute adrenal weights of SHRs tended to be higher than in Lewis rats; taken with the observation that the former strain weighs less than the latter one (thus confirming previous observations: Ramos et al. 1997, Berton et al. 1998), this tendency reached significance when relative adrenal weights were considered. In turn, it suggests that adrenal stimulation by ACTH is increased in SHRs compared with Lewis rats. However, compared with the values previously reported in undisturbed SHRs and Lewis rats (Berton et al. 1998), SHR displayed a 12 -fold and Lewis a 2 -fold increase in plasma CORT. Basal values of plasma CORT in untrained control Lewis were within the values observed in a context of social stress in this strain but remained 5-fold above in SHRs (Berton et al. 1998). These results suggest that under our experimental conditions, untrained rats were significantly stressed. This may be caused by the daily exposure to a novel environment as controls of each strain were gently handled and moved into a novel cage. Such a paradigm was used to obtain appropriate controls to the exercising rats, e.g. change in environment without exercise. Our study, using SHRs and Lewis rats, extends prior evidence for SHRs being more reactive than normotensive rats, e.g. Wistar-Kyoto and Sprague-Dawley rats, to a variety of stressful stimuli, including in their behavioural and HPA axis responses (McMurtry \& Wexler 1981, Kräuchi et al. 1983, Gentsch et al. 1987, Söderpalm 1989). For example, our data in Lewis rats are in agreement with previous reports demonstrating a hyporesponsiveness of Lewis HPA axis to a wide range of acute (Glowa et al. 1992, Dhabhar et al. 1993,
Chaouloff et al. 1995) and chronic stress situations (Gomez et al. 1998).

Previous reports have indicated that Trp levels were higher in the midbrain of SHRs, compared with those of Lewis rats (Chaouloff et al. 1997, Berton et al. 1998). In the present study, such a difference was confirmed and extended from midbrain serotonergic cell bodies to striatal and hippocampal serotonergic nerve terminals. Interestingly, the genetic difference in brain Trp availability was associated with a strain-dependent difference in circulating $\operatorname{Trp}$ levels. Whether the latter difference underlies the former one is a possibility that merits consideration. However, because brain Trp availability may depend on other variables (e.g. bloodbrain batrier permeability, blood neutral amino acids, blood free Trp: see Chaouloff 1993) it is premature to draw any conclusion as to the mechanisms underlying the strain-dependent difference in brain Trp levels.

Except for a strain effect on striatal 5-HT levels, neither 5-HT nor 5-HIAA were found to differ between SHRs and Lewis rats, a finding that fits with our observation of a lack of strain differences in midbrain and hippocampus 5-HT turnover, as assessed by the measurement of 5-hydroxytryptophan accumulation in NSD-1015-pretreated rats (Kulikov et al. 1997).

\section{Effects of chronic exercise or acute stress in SHRs and Lewis rats}

One element of the differential response between both strains of rats was the effect of chronic exercise on their weight evolution. As early as the third week of exercise training and thereon, SHRs displayed significantly less weight gain than their Lewis counterparts. Actually, the 
effect of exercise training on weight balance is controversial. Some studies have shown that exercise training such as treadmill running leads to hypophagia, and in turn weight loss (Rivest \& Richard 1990). Others, however, have found no exercise effect on body mass using similar (Overton et al. 1991, Watanabe et al. $1992)$ or more intense exercise programmes $(60 \mathrm{~min}$ at $30 \mathrm{~m} \mathrm{~min}^{-1}$ for 6 consecutive weeks; White-Welkley et al. 1995).

The observation of a precocious and important exercise-induced weight loss in SHRs strengthens the hypothesis that the two strains are differentially affected by exercise training. The precocious weight loss in SHRs may suggest that the exercise training was more intensive in SHRs compared with Lewis rats. We used a standard paradigm of exercise training that has been previously shown to elicit $50-60 \%$ of the SpragueDawley rat's $V_{O_{2 \max }}$ (Bedford et al. 1979). However, in that study which compared five inbred rats, Bedford et al. have demonstrated an interstrain $V_{\mathrm{O}_{2} \max }$ difference with a decreased $\mathrm{VO}_{2 \max }$ in SHRs, compared with Sprague-Dawley rats, thereby suggesting that in our study SHRs may have been overtrained compared with Lewis rats. Alternatively, SHRs have been shown to run long distances in another exercise paradigm, e.g. spontaneous wheel running (Hoffmann et al. 1990, Jonsdottir et al. 1998) indicating that SHRs display significant endurance capacities. Moreover, (i) using more intense exercise programmes than ours, WhiteWelkley et al. (1995) have not found any effect on body weight on female Sprague--Dawley rats and (ii) increases in basal CORT values have been reported in overtrained subjects, e.g. an observation that differs from ours where a significant decrease in plasma CORT values after 8 weeks of exercise training was observed in SHRs compared with Lewis rats (see below). With regard to Lewis rats, the possibility that this strain resists to some physiological consequences of training (e.g. corticotropic ones: see below) must be advanced. It is noteworthy that Lewis rats underwent a training protocol that did not differ from that of SHRs, thereby excluding differences in the distances covered during the training programme as a source of the aforementioned interstrain variability. However, to explore the intrinsic effects of training in Lewis rats, compared with SHRs, experiments devoted to the analysis of muscular oxidative enzyme activities in sedentary and trained SHRs and Lewis rats are already in progress in our laboratory.

The activity of the HPA axis was also differentially affected by exercise training. Forty-eight hours after the end of the exercise training program, a $40 \%$ decrease in basal plasma CORT was noticed in the SHR strain compared with untrained rats (albeit without statistical significance), whereas a significant basal plasma CORT increase was observed in the Lewis strain. This discrepancy concerning the effects of chronic exercise on HPA activity (i) between both strains and (ii) within the same strain compared with the effect of environmental handling, may appear puzzling at first. Focusing on Lewis rats, one might suppose that environmental handling was a weaker intensity stress than repeated daily forced muscle exercise. Nevertheless, these results depict a hyporesponsiveness of the HPA axis in this strain as plasma CORT remained lower than that of SHRs in all cases. The HPA axis hyporeactivity of Lewis rats seems to be located at the adrenocortical level as the blunted CORT response to chronic exercise, compared with SHRs, is not caused by a defect in basal ACTH release. It has been previously reported that unstressed Lewis rats have less adrenocortical cells than Wistar rats (in spite of a similar adrenal weight) as well as blunted in vitro and in vivo adrenocortical responses to exogenous ACTH (Oitzl et al. 1995). This reduced adrenocortical sensitivity to $A C T H$ in the adult male Lewis rat is in accordance with the results of Gomez et al. (1996) who reported a CORT hyporesponsiveness to chronic immobilization stress, that may be linked to adrenal hyposensitivity to ACTH. In our study, the CORT/ACTH ratios were decreased in Lewis rats compared with SHRs, this being true both in trained and untrained rats. On the other hand, one cannot exclude the hyperfunctioning of the adrenals in SHRs as suggested by Gomez et al. (1998).

Whatever the mechanism of the HPA axis hyperactivity in SHRs, exercise training was found to diminish the HPA axis hyperactivity in SHRs. These findings may thus be relevant to the study of the mechanisms underlying the protective effects of exercise training on the deleterious hyperresponsiveness of HPA axis to behavioural stress. Recent studies suggest that excessive activity of glucocorticoid hormones is deleterious and associated with increased cardiovascular risk factors (Walker 1996). Moreover, it has been demonstrated that adrenalectomy prevents the development of hypertension in SHRs (Hashimoto et al. 1989). Corticosterone teplacement restored hypertension, which suggests that CORT is essential for the development of hypertension in SHRs. However, the former authors (Hashimoto et al. 1989) have demonstrated that hypertension did not depend entirely on peripheral corticosterone levels and suggested that SHRs presented an exaggerated response of blood pressure to corticosterone. In humans, Walker et al. (1998) have recently suggested that enhanced cortisol activity may be an element of the familial predisposition to high blood pressure. Lastly, exercise training is known, in normotensive rats (Wistar Kyoto), to attenuate stress-induced hypertension (Cox et al. 1985). Under these conditions, SHRs may be a relevant model to. 
humans where increased cardiovascular and HPA axis reactivities to behavioural stress are to be found.

Lastly, in untrained rats, the effect of intense stress such as 1 -h immobilization may appear controversial with regard to the aforementioned results. Indeed, immobilization stress significantly stimulated the HPA axis in both strains, as evidenced by the increase in plasma ACTH concentrations. Consequently, plasma CORT also increased significantly, with plasma CORT levels of Lewis reaching those of SHRs. In untrained SHRs, no change in plasma CORT could be observed. ACTH levels further increased, a discrepancy that lies into a yet maximal adrenal secretory capacity. There are several mechanisms that may account for such a discrepancy between the respective responses of the HPA axis to chronic exercise on the one hand and acute immobilization stress on the other hand, including the existence of $\mathrm{ACTH}$-independent mechanisms (Holzwarth et al. 1987).

It has been reported that treadmill endurance training may affect central 5-HT levels (Brown et al. 1979). In addition, a 7-week wheel running exercise was found to elicit region-dependent decreases in 5-HT turnover (Hoffmann et al. 1994). However, in our hands, 8 weeks of training on a treadmill were found to be ineffective on central Trp, 5-HT and 5-HIAA levels in Wistar rats (Chaouloff et al. 1987). The present study confirms such a lack of effect of endurance training. On the other hand, central 5-HT synthesis and metabolism have been reported to be stimulated by acute immobilization (for a review: Chaouloff 1993). This was also true herein as immobilization increased Trp and 5-HIAA in all brain regions (albeit with different intensities) of SHRs and Lewis rats, a change that was associated with a decrease in blood Trp levels. The latter observation, which is in line with past data, is noteworthy as stress-induced decreases in blood $\operatorname{Trp}$ levels may reflect sympathetic hyperactivity (Chaouloff 1993). Then, the observation that both strains displayed to similar extents such a stress-elicited decrease in blood Trp levels indicate that the aforementioned strain-dependent sensitivity of the HPA axis may be unique. Interestingly, immobilization-induced increases in brain Trp levels, and which are accounted for by an increase in blood-brain barrier permeability (Chaouloff 1993) were greater in SHRs, compared with Lewis rats. Whether such a genetic difference lies on differences in blood-brain permeability (Werber et al. 1990) remains however, to be shown.

\section{Effects of endurance training on the responses to acute stress in SHRs and Lewis rats}

In Lewis rats, exercise training increased the effect of 1-h immobilization on plasma ACTH concentrations without further increasing plasma CORT levels.
Previous studies have emphasized the influence of an heterotypic stress in chronically stressed rats (Watanabe et al. 1991, 1992), demonstrating that the secretion of CORT by adrenal cells could be augmented if the adrenal had been previously primed by ACTH (such as that elicited by prior chronic stress). The absence of any increase in CORT levels although ACTH stimulation increased lends further support to the above-mentioned hypothesis according to which Lewis rats display a decreased adrenocortical sensitivity to ACTH.

An overview of midbrain, striatum and hippocampus Trp levels suggests that the amplitude of immobilization-elicited increases in Trp levels was decreased by training in SHRs but increased by training in Lewis rats, this being especially true in the midbrain and the hippocampus. Thus, with regard to brain Trp availability, training amplified (Lewis rats) or diminished (SHRs) the stimulatory effect of immobilization stress. This could not be accounted for by changes in blood Trp levels as in Lewis rats, immobilization-elicited reduction in blood Trp was slightly diminished in trained rats, compared with untrained rats. Clearly, some additional factors, e.g. other than blood Trp, played a key role in the aforementioned genetic variability in brain Trp availability during immobilization. With regard to the effects of training on immobilization-elicited increases in tissue 5-HIAA levels (which arise from parallel changes in tissue Trp levels, and possibly Trp hydroxylase hyperactivity: Chaouloff 1993), the picture was far from being clear. In midbrain, training amplified the stimulatory effect of immobilization in both strains, thereby suggesting that compensatory changes (involving possibly Trp hydroxylase activity) took place so that the genetic differences in Trp availability were masked. In the hippocampus, besides the overall stimulatory effect of immobilization in 5-HIAA levels, it could be observed that training amplified (Lewis) or diminished (SHRs) the amplitude of the latter stimulation; in the striatum, however, such a strain-dependent profile was not observed. Whether these regional differences reflect specific effects of immobilization and training with regard to the dorsal raphe nucleus (where striatal serotonergic nerves come from) as opposed to the median raphe nucleus (where a majority of hippocampal serotonergic nerves come from) is unknown.

\section{REFERENCES}

Armario, A., Gavalda, A. \& Marti, J. 1995. Comparison of the behavioural and endocrine response to forcd swimming stress in five inbred strains of rats. Psychoneuroendocrinology 20, 879-890.

Armstrong, R.B., Laughlin, M.H., Rome, L. \& Taylor, C.R. 1983. Metabolism of rats running up and down an incline. J Appl Pbysiol 55, 518-521. 
Barbato, J.C., Koch, L.G., Darvish, A., Cicila, G.T., Metting, P.J. \& Britton, S.L. 1998. Spectrum of aerobic endurance running performance in eleven inbred strains of rats. J Appl Pbysiol 85, 530-536.

Bedford, T.G., Tipton, C.M., Wilson, N.C., Oppliger, R.A. \& Gisolfi, C.V. 1979. Maximum oxygen consumption of rats and its changes with various experimental procedures. J Appl Physiol 47, 1278-1283.

Berton, O., Aguerre, S., Sarrieau, A., Mormède, P. \& Chaouloff, F. 1998. Differential effects of social stress on central serotonergic activity and emotional reactivity in Lewis and spontaneously hypertensive rats. Neuroscience 82, 147-159.

Brown, B.S., Payne, T., Kim, C., Moore, G., Krebs, P. \& Martin, W. 1979. Chronic response of rat brain norepinephrine and serotonin levels to endurance training. $J$ Appl Pbysiol 46, 19-23.

Chaouloff, F. 1993. Physiopharmacological interactions between stress hormones and central serotonergic systems. Brain Res Rev 18, 1-32.

Chaouloff, F., Baudrie, V. \& Coupry, I. 1994. Effects of chlorisondamine and restraint on cortical $\left[{ }^{3} \mathrm{H}\right]$ ketaserin binding, $5-\mathrm{HT}_{2 \mathrm{~A}}$ receptor-mediated head shakes, and behaviours in models of anxiety. Neuropharmacology 33, 449-456.

Chaouloff, F., Berton, O., Aguerre, S., Hay, M. \& Mormède, P. 1997. Effects of food deprivation on midbrain $5-\mathrm{HT}_{1 \mathrm{~A}}$ autoreceptors in Lewis and SHR rats. Neuropharmacology 36, 483-488.

Chaouloff, F., Elghozi, J.L., Guezennec, Y. \& Laude, D. 1985. Effects of conditioned running on plasma, liver and brain tryptophan and on brain 5-hydroxytryptamine metabolism of the rat. Br J Pharmac 86, 33-41.

Chaouloff, F., Kulikov, A., Sarrieau, A., Castanon, N. \& Mormède, P. 1995. Male Fisher 344 and Lewis rats display differences in locomotor reactivity, but not in anxietyrelated behaviours: relationship with the hippocampal serotonergic system. Brain Res 693, 169-178.

Chaouloff, F., Laude, D., Serrurrier, B., Merino, D., Guezennec, Y. \& Elghozi, J.L. 1987. Brain serotonin response to exercise in the rat: the influence of training duration. Biogenic Amines 4, 99-106.

Cox, R.H., Hubbard, J.W., Lawler, J.E., Sanders, B.J. \& Mitchell, V.P. 1985. Exercise training attenuates stress-induced hypertension in the rat. Hypertension 7 , 747-751.

Dhabhar, F.S., McEwen, B.S. \& Spencer, R.L. 1993. Stress response, adrenal steroid receptor levels and corticosteroidbinding globulin levels - a comparison between Sprague-Dawley, Fischer 344 and Lewis rats. Brain Res 616, 89-98.

Duclos, M., Corcuff, J.-B., Rashedi, M., Fougère, V. \& Manier, G. 1996. Trained versus untrained men: different immediate post-exercise responses of pituitary adrenal axis. A preliminary study. Eur J Appl Physiol 73, 427-433.

Gentsch, C., Lichtsteiner, M. \& Feer, H. 1987. Open field and elevated plus-maze: a behavioural comparison between spontaneously hypertensive (SHR) and Wistar-Kyoto (WKY) rats and the effects of chlordiazepoxide. Bebav Brain Res 25, 101-107.
Glowa, J.R., Geyer, M.A., Gold, P.W. \& Sternberg, E.M. 1992. Differential startle amplitude and corticosterone response in rats. Neuroendocrinology 56, 719-723.

Gomez, F., De Kloet, R. \& Armario, A. 1998. Glucocorticoid negative feedback on the HPA axis in five inbred rat strains. Am J Physiol 274, R420-R427.

Gomez, F., Lahmame, A., De Kloet, E.R. \& Armario, A. 1996. Hypothalamic-pituitary-adrenal responses to chronic stress in five inbred rat strains: differential responses are mainly located at the adrenocortical level. Neuroendocrinology 63, 327-337.

Hagberg, J.M., Ferrell, R.E., McCole, S.D., Wilund, K.R. \& Moore, G.E. 1998. $\mathrm{VO}_{2} \max$ is associated with $\mathrm{ACE}$ genotype in postmenopausal women. J Appl Physiol 85, 1842-1846.

Hashimoto, K., Makino, S., Hirasawa, R. et al. 1989. Abnormalities in the hypothalamo-pituitary-adrenal axis in spontaneously hypertensive rats during development of hypertension. Endocrinology 125, 1161-1167.

Henriksson, J., Svedenhag, J., Richter, E.A., Christensen, N.J. \& Galbo, H. 1985. Skeletal muscle and hormonal adaptation to physical training in the tat: role of the sympatho-adrenal system. Acta Pbysiol Scand 123, 127-138.

Hoffmann, P., Elam, M., Thoren, P. \& Hjorth, S. 1994. Effects of long-lasting voluntary running on the cerebral levels of dopamine, serotonin and their metabolites in the spontaneously hypertensive rat. Life Sci 54, 855-861.

Hoffmann, P., Terenius, L. \& Thoren, P. 1990. Cerebrospinal fluid immunoreactive beta-endorphin concentration is increased by voluntary exercise in the spontaneously hypertensive rat. Regul Pept 28, 233-239.

Holzwarth, M.A., Cunningham, L.A. \& Kleitman, N. 1987. The role of adrenal nerves in the regulation of adrenocortical functions. Ann NY Acad Sci 512, 449-464.

Jonsdottir, I.H., Jungersten, L., Johansson, C., Wennmalm, A., Thoren, P. \& Hoffmann, P. 1998. Increase in nitric oxide formation after chronic voluntary exercise in spontaneously hypertensive rats. Acta Pbysiol Scand 162, 149-153.

Koch, L.G., Meredith, T.A., Fraker, T.D., Metting, P.J. \& Britton, S.L. 1998. Heritability of treadmill running endurance in rats. Am J Physiol 275, R1455-R1460.

Kräuchi, K., Wirz-Justice, A., Willener, R., Campbell, I.C. \& Feer, H. 1983. Spontaneous hypertensive rats: behavioural and corticosterone tesponse depend on circadian phase. Physiol Behav 30, 35-40.

Kregel, K.C., Overton, J.M., Seals, D.R., Tipton, C.M. \& Fisher, L.A. 1990. Cardiovascular responses to exercise in the rat: role of corticotropin-releasing factor. J Appl Pbysiol 68, 561-567.

Kulikov, A., Aguerre, S., Berton, O., Ramos, A., Mormède, P. \& Chaouloff, F. 1997. Central serotonergic systems in the SHR and LEW rat strains that differ in the elevated plusmaze test of anxiety. J Pharmac Exp Ther 281, 775-784.

Luger, A., Deuster, P., Kyle, S.B. et al. 1987. Acute hypothalamic-pituitary-adrenal responses to the stress of treadmill exercise. New Engl J Med 316, 1309-1315.

McMurtry, J.P. \& Wexler, B.C. 1981. Hypersensitivity of spontaneously hypertensive rats (SHR) to heat, ether, and immobilization. Endocrinology 108, 1730-1736. 
Oitzl, M.S., Van Haarst, A.D., Sutanto, W. \& De Kloet, E.R. 1995. Corticosterone, brain mineralocorticoid receptors (MRs) and the activity of the hypothalamic-pituitaryadrenal (HPA) axis: the Lewis rat as an example of increased central MR capacity and hyporesponsive HPA axis. Psychoneuroendocrinology 6, 655-675.

Overton, J.M., Kregel, K.C., Davis-Gorman, G., Seals, D.R., Tipton, C.M. \& Fisher, L.A. 1991. Effects of exercise training on responses to central injection of CRF and noise stress. Physiol Behav 49, 93-98.

Petrides, J.S., Gold, P.W., Mueller, G.P. et al. 1997. Marked differences in functioning of the hypothalamic-pituitaryadrenal axis between groups of men. J Appl Physiol 82, 1979-1988.

Ramos, A., Berton, O., Mormède, P. \& Chaouloff, F. 1997. A multiple-test study of anxiety-related behaviours in six inbred rat strains. Behav Brain Res 85, 57-69.

Rivest, S. \& Richard, D. 1990. Hypothalamic paraventricular nucleus lesions do not prevent anorectic effect of exercise in male rats. Am J Pbysiol 259, R579-R584.

Söderpalm, B. 1989. The SHR exhibitless 'Anxiety' but increased sensitivity to the anticonflict effect of clonidine compared to normotensive controls. Pharmacol Toxicol 65, 381-386.

Sternberg, E.M., Glowa, J.R., Smith, M.A. et al. 1992.

Corticotropin releasing hormone related behavioural and neuroendocrine responses to stress in Lewis and Fisher rats. Brain Res 570, 54-60.

Walker, B.R. 1996. Abnormal glucocorticoid activity in subjects with risk factors for cardiovascular disease. Endocr Res 22, 701-708.

Walker, B.R., Philips, D.I.W., Noon, J.P. et al. 1998. Increased glucorticoid activity in men with cardiovascular tisk factors. Hypertension 31, 891-895.

Watanabe, T., Morimoto, A., Sakata, Y., Tan, N., Morimoto, K. \& Murakami, N. 1992. Running training attenuates the ACTH responses in rats to swimming and cage-switch stress. J Appl Physiol 73, 2452-2456.

Watanabe, T., Morimoto, K., Sakata. Y., Wada. M. \& Murakami. N. 1991. The effect of chronic exercise on the pituitary-adrenocortical response in conscious rats. $J$ Pbysiol 439, 691-699.

Werber, A.H., Fitch-Burke, M.C. \& Tobin, P.C. 1990. Hypertension and cerebral arteries: relationship to disruption of the blood-brain barrier. Clin Exp Hypertens 12, 243-265.

White-Welkley, J.E., Bunnel, B.N., Mougey, E.H., Meyerhoff, J.L. \& Dishman, R.K. 1995. Treadmill exercise training and estradiol differentially modulate hypothalamic-pituitaryadrenal cortical responses to acute running and immobilization. Pbysiol Bebav 57, 533-540. 